\title{
MATERNAL FOLIC ACID AND RISK OF CHILDHOOD AUTISM
}

Researchers from the Norwegian Institute of Public Health, Oslo; Institute of Child Health, London, UK; and other centers in Norway, UK, and the US examined the association between maternal use of prenatal folic acid supplements ( 4 weeks before to 8 weeks after start of pregnancy) and risk of autistic spectrum disorders in children derived from the population-based, prospective Norwegian Mother and Child Cohort Study (MoBa). The age-range at end of follow-up was 3.3 - 10.2 years (mean, 6.4 years). The total daily maternal folate intake varied widely from 62-5673 ug (mean, $497 \mathrm{ug} /$ day).

At end of follow-up, a total of 270 children $(0.32 \%)$ were diagnosed with ASDs: 114 autistic disorder, 56 Asperger syndrome, 100 PDD-NOS. Autistic disorder was diagnosed in $0.1 \%$ of children exposed to folic acid compared with $0.21 \%$ in those unexposed. The adjusted odds ratio for autistic disorder in children of folic acid users was 0.61 . In contrast, children of mothers who supplemented their diet with fish oil showed no association with autistic disorder. The findings do not establish causality but they do add support to prenatal folic acid diet supplementation. (Suren P, Roth C, Bresnahan M, et al. Association between maternal use of folic acid supplements and risk of autism spectrum disorders in children. JAMA 2013 Feb 13;309(6):570-7). (Response: Pal Suren MD, MPH, Norwegian Institute of Public Health, PO Box 4404, Nydalen, N-0403 Oslo, Norway. E-mail: pal.suren@fhi.no).

COMMENT. Maternal folic acid supplementation during pregnancy, widely known to reduce the risk of neural tube defects in children, is now shown to be associated with a lower risk of childhood autism. It has also been linked to a lower risk of severe language delay at age 3 years, a study also conducted in Norway (Roth C, et al. JAMA 2011 Oct 12;306(14):1566-73).

\section{GESTATIONAL AGE AT BIRTH AND RISK OF AUTISM}

The association between gestational age (GA) at birth and the risk of autistic spectrum disorder (ASD) in 218,110 singleton live births between 1998 and 2004 in Alberta, Canada was studied at each completed week of gestation $(\mathrm{GA}<23$ weeks vs $>23$ weeks to $<43$ weeks vs $>43$ weeks, in 1-week increments). A gradual increased risk of developing ASD was noted with shorter gestation. Cutoffs between 29 and 40 weeks clearly denoted an elevated risk of ASD compared with longer gestation, and the risk increased with earlier GA cutoff. The association was independent of sex and fetal growth measures. (Leavey A, Zwaigenbaum L, Heavner K, Burstyn I. Gestational age at birth and risk of autism spectrum disorders in Alberta, Canada. J Pediatr 2013 Feb;162(2):361-8). (Reprints: Igor Burstyn PhD, Drexel University, Philadelphia, PA. Email: igor.burstyn@drexel.edu). This study confirms the findings in an earlier smaller sample size population (Gillberg C, Gillberg IC. Infantile autism: a total population study of reduced optimality in the pre-, peri- and neonatal period. J Autism Dev Disord 1983 Jun;13(2):153-66). 